\title{
Telematic mediations: Comments on hyper-relationships
}

\author{
Keywords \\ Hyper-relations, Software Studies, Technodiversity
}

This abstract is a synthesis of a theoretical exercise applied to an ongoing series of collages whose process began in 2021. The work, grounded in a critique of the hyper-relationships of a codified ecosystem and the lack of what Hui (2020) calls technodiversity, is a direct result of doctoral-level artistic experimentation. Deprived of physical contact and exchanges due to the pandemic, we work and continue to do so at a distance. Exercising the poetics under a condition that can be described as exiled, we communicate regularly to extract, in the somewhat hermetic relationship of the virtual, sparks of what one expects from the other. This dynamic, representative of the condition expressed in the series, echoes the magic of collaboration by associating synergic experimentation with learning, allowing the establishment of an experience of shock or surprise, which is nevertheless always exciting (KIDNER, 2018). Derived from a phenomenological reduction movement (FARINA, 2008) that transforms traditional images into technoimages, the series' plastic decision seeks to create "static performances" drawn from the contemporary way of life mediated by ubiquitous interfaces. Such a strategy, articulated under the software theory - "the invisible glue that binds everything together" (MANOVICH, 2008) - is what allows for the realization of embodiment as "an opportunity to integrate physical and digital worlds" (JEON, 2019). The work is characterized by an analysis of the presence of bodies paradoxically united in absence. The distance between beings, verified in the impossibility of being, brings the individuals together in a metaphysical experience only conceivable in the telematic sphere. Associations triggered by elements such as sky and sea provide the basis for thinking about clouds and waves. Employed as denaturalized metaphors of nature, the elements emerge as enablers of connections between distant, disembodied, and dematerialized bodies. The series stresses the growing social movement that "moves, clearly, from things to information" (FLUSSER, 2008), evidencing the tangential nature of physicality. The phenomenological reduction is observed in the dismissal of spatiality and allows us to think under the terms of a post-real aesthetic, which does not ignore the existence of the real, but transports its conditions of experimentation to a scale of impalpable nature. Thinking about human relations under the virtualized dimension of the sensible is an exercise of abstraction made easier by technological homogeneity, a condition that establishes itself in the maintenance of protocols that can be experienced on a planetary scale. This means that communicators around the world can interact based on common resources (software), namely developed by transnational companies that hold technological sovereignty. The downside, paradoxically, is to be found in the clarity of these very assumptions. The methodological exercise as articulated here is applied to the monotechnology recognition that limits the possibilities of a creative technodiversity $(\mathrm{HUI}, 2020)$ and reduces digital experience to a dangerous hegemonic dependency. More like a plastic diagnosis of the software-mediated world and less like a dossier of major solutions, the series proposes thoughts regarding problems that resound from the telematic experience and addresses the socially accepted technological hegemony. 